\title{
Los investigadores sociales y su relación con los campesinos en Colombia: ¿tacto o indiferencia?
}

\section{María Teresa Matijasevic y Alexander Ruiz Silva}

Fecha de recepción: 11 de marzo de 2013

Fecha de aprobación: 7 de octubre de 2013

\begin{abstract}
Resumen
¿Cuál ha sido el compromiso ético y político de los investigadores sociales en Colombia respecto a los campesinos?, ¿qué discursos han predominado en relación con ellos? El presente artículo aporta algunos elementos de discusión en torno a estos interrogantes, partiendo especialmente de estados del arte realizados por autores colombianos sobre el conocimiento producido en las últimas décadas respecto a los campesinos, el sector rural y los jóvenes de las zonas rurales. Pese a la presencia de importantes investigadores en el país dedicados a esta temática, el análisis de la producción académica en torno a los campesinos permite identificar un interés insuficiente en la problemática de este grupo por parte de la generalidad de investigadores sociales colombianos; así como la tendencia a privilegiar análisis de carácter económico, a los que se ha sumado, en las últimas décadas, un importante número de estudios enfocados en el conflicto armado y en el desplazamiento forzado, dejando de lado -o abordando de manera insuficiente- importantes dimensiones de la vida campesina. Además de la aproximación a los estudios realizados, el artículo llama la atención sobre la necesidad de ampliar los marcos a partir de los cuales se analiza el compromiso ético y político de la investigación social.
\end{abstract}

Palabras clave: campesinos, rural, jóvenes rurales, investigación social, investigación rural.

Abstract
What has been the ethical and political commitments of social
researchers to peasants in Colombia? What are the prevailing discourses
in this relationship? This article provides some elements to discuss
these questions. It starts from the state of affairs of the production of
knowledge on peasants, the rural sector and young people living in rural
areas as studied by Colombian researchers in the last decades. In spite
of the fact that there are important researchers studying this topic, the
analysis of their academic production reveals an insufficient interest in
the problematic of this social group by Colombian researchers. The study
also reveals a tendency towards the economic analysis with emphasis on
studies focused on the armed conflict and forced displacement rather
than the analysis of fundamental dimensions of the life as a peasant. 
This article also draws attention to the need to expand the theoretical frameworks for the analysis of the ethical and political commitments of social research.

Keywords: peasants; rural, rural young people; social research; rural research.

\section{Introducción}

El mundo de la ciudad está en peligro de sucumbir a una falsa creencia corruptora. Una impertinencia muy ruidosa y muy activa y muy delicada parece, a menudo, preocuparse por el mundo y la existencia del campesino. Pero con ello se niega precisamente lo que ahora sólo hace falta: mantener la distancia de la existencia campesina; abandonarla -ahora más que nunca- a su propia ley; ;fuera las manos!; para no arrastrarla en una falsa habladuría de literatos sobre lo popular y el amor a la tierra. El campesino ni quiere ni necesita en ningún caso esta exagerada amabilidad ciudadana. Lo que ciertamente necesita y quiere es el tacto reservado respecto a su propio ser y a su independencia (Heidegger, 1963).

Más allá de la lectura contextual e histórica que exigiría el epígrafe de Heidegger -no realizada aquí-, su planteamiento invita a reflexionar sobre las formas de interacción que han predominado en el país entre investigadores y campesinos y sobre el sentido que adquieren las distintas maneras de producir conocimiento, considerando la problemática campesina y la historia de exclusión de este grupo social.

Reconociendo la necesidad de otras formas de exploración en las que los propios campesinos serían indispensables, este artículo pretende aportar elementos para la discusión en torno a dos interrogantes: ¿Cuál ha sido el compromiso ético y político de los investigadores sociales en Colombia respecto a los campesinos?, ¿qué discursos han predominado en relación con ellos? Como recurso para aproximarse a estas reflexiones se parte fundamen- 
talmente de los estados del arte realizados por algunos autores colombianos en torno al conocimiento producido en las últimas décadas en Colombia respecto a los campesinos, pero también al sector rural y a los jóvenes rurales .

Partir de estos estados del arte tiene la ventaja de facilitar una visión de conjunto, ya depurada por especialistas en el tema, pero naturalmente no agota la totalidad de conocimientos producidos en torno a esta población, no sólo por las perspectivas disciplinares que pudieron privilegiarse, sino también por los períodos analizados y las áreas temáticas abordadas. Pese a estas limitaciones, los hallazgos de estos estudios son suficientemente reveladores de algunas de las principales tendencias de la investigación social que se ha realizado en el país en relación con los campesinos, permitiendo abocar, al menos parcialmente, las reflexiones propuestas. Éstas son retomadas en la segunda parte del artículo, una vez presentado un balance general sobre las tendencias identificadas en los mencionados estados del arte.

\section{La investigación rural y campesina en Colombia}

Un primer elemento que debe considerarse al emprender la aproximación propuesta es la posibilidad de distinguir entre estudios rurales y estudios urbanos, separación que es problematizada, al menos, desde tres perspectivas. La primera de ellas, señalada por Ibáñez, cuestiona el origen mismo de esta separación. A su juicio, la distinción es creada desde una perspectiva urbana: "El explotador pertenece al lugar desde el que se traza la raya" (Ibáñez, 1991, 96). Refiriéndose concretamente a un campo disciplinar, considera que la separación entre la sociología urbana y rural disimula y refuerza la relación de dominación existente entre la ciudad y el campo, relación que contribuyen a mantener los sociólogos rurales al aceptar autodenominarse como tales.

Una segunda perspectiva se encuentra en Fals Borda (1963), quien en los primeros desarrollos sistemáticos de la sociología en 
Colombia llamó la atención sobre la necesidad de que la sociología rural conservara la alianza conceptual y teórica con la sociología como ciencia madre: "A fin de comprender mejor los grupos campesinos debe lograrse el conocimiento de sus relaciones con los grupos de la ciudad; e igualmente, muchos problemas urbanos se enraízan en el movimiento emigratorio de las comunidades rurales, y por tanto la ruralidad como factor no puede ser excluido" (154). La preocupación de Fals Borda se remitía pues a la necesidad de superar separaciones intradisciplinares que pudieran dificultar una comprensión integrada de los problemas sociales.

Parte importante de la problematización actual sobre esta separación gira alrededor de la dificultad en ciencias sociales de hacer referencia a contextos y a poblaciones específicamente rurales o campesinas. Se podría considerar ésta como una tercera perspectiva, observada en autores como Baigorri (1995) y Llambí (2010), quienes aducen que la sociología rural, tal como fue concebida en sus inicios, carece de sentido actualmente, dada la superación de la dicotomía urbano-rural y la existencia de experiencias sociales desterritorializadas que exigen una nueva mirada. Mientras Llambí (2010) ve necesaria la construcción de una nueva sociología rural a partir de un trabajo interdisciplinar, que atienda las heterogeneidades y las interacciones entre el contexto biofísico, los sistemas de producción y las sociedades rurales, Baigorri (1995) propone una sociología de la urbanización, cuyo objeto sería el análisis de los procesos de cambio que propician la integración de los espacios rurales en la urbe global. Más que una deconstrucción de la dicotomía urbano-rural se observa, en este discurso, una predominancia de lo urbano sobre lo rural, en el que lo segundo sería apenas un residuo de lo primero.

A excepción del planteamiento de Fals Borda, no se encuentra en los textos revisados sobre la investigación rural en Colombia una problematización explícita sobre la separación entre los estudios urbanos y los rurales. Cubides (1998), en un análisis am- 
plio sobre la sociología en Colombia, considera que la dicotomía entre el mundo rural y el mundo urbano -propia de la primera etapa de esta disciplina en el país- fue abandonada, dando lugar a sus imbricaciones y continuidades. No son del todo claras, sin embargo, las implicaciones de la supuesta superación de esta dicotomía en los estudios llevados a cabo actualmente en el país.

\subsection{Estudios sobre la ruralidad}

Fals Borda identificó, en 1963, tres etapas en el desarrollo de la sociología rural en Colombia. Una inicial, a la que denominó filosófica, fuertemente influida por las teorías sociológicas europeas, de la cual se derivaron descripciones detalladas de los grupos campesinos y de los procesos rurales, visión ésta realizada por fuera de la estructura conceptual sociológica. Este trabajo resalta, en el caso de Colombia, las obras de Luis López de Mesa, médico especializado en psiquiatría y psicología, autor de distintas obras a las que acuñó el nombre de sociología, en las que se destaca, entre otros aspectos, su interés por la identidad de la sociedad colombiana. Una segunda etapa, de transición, caracterizada por el predominio de la sociología empírica, promovida por la presencia en América Latina de varios sociólogos rurales, como consultores adjuntos a las Embajadas de los Estados Unidos después de la segunda guerra mundial. Aquí se destaca, entre otros, a Lynn Smith, quien después fuese profesor del mismo Fals-Borda y quien realizó el primer estudio rural en Colombia -en la comunidad cundinamarquesa de Tabio- utilizando un marco de referencia sociológico e introduciendo el uso de encuestas y entrevistas guiadas, con la colaboración de dos abogados colombianos, Justo Díaz Rodríguez y Luis Roberto García. De acuerdo con Fals Borda (1963), el método utilizado había sido esbozado por los sociólogos europeos y trasladado a Norteamérica, donde floreció, mientras los conflictos bélicos frustraron en Europa los esfuerzos similares de los científicos sociales. De ahí que este autor considere que haya existido menor influencia europea que norteame- 
ricana en la sociología de la vida rural en Colombia. Por último, una etapa de formación, caracterizada por la construcción de la sociología desde una perspectiva cultural latinoamericana, cuyo surgimiento se vio facilitado por el impulso inicial dado por los sociólogos europeos y norteamericanos a los estudios rurales en América Latina, la formación de sociólogos en el país y la dinámica misma de la sociedad colombiana, que exigía un nuevo tipo de sociología y que permitió la creación de diversas escuelas e institutos de investigación social.

Machado (2004), quien analiza los vínculos existentes entre la academia colombiana y el sector rural a partir de los años 50 del siglo pasado, presenta un panorama similar al esbozado por Fals Borda (1963) en las dos últimas etapas, no circunscrito estrictamente a la sociología. En lo que puede asimilarse a la etapa de transición, Machado (2004) refiere que la producción académica sobre el sector rural se inició a partir del informe de Lauchlin Currie en 1950, al que le siguieron distintos análisis y recomendaciones de entidades internacionales orientados a la modernización de la agricultura y a la reforma agraria. Fals Borda da cuenta también, en 1963, de la fuerte presión que se estaba ejerciendo en Colombia y en América Latina sobre los científicos sociales para que prestaran atención a los problemas rurales, tal como sucedió en Estados Unidos a principios del siglo pasado y que dio lugar en ese país a la creación de la sociología rural.

En estrecha relación con la etapa de formación planteada por Fals Borda (1963), Machado (2004) refiere el surgimiento en los años 60 de autores colombianos cuya producción se aparta de la influencia de los pensadores extranjeros y de los intereses de los organismos internacionales. Ubica en este grupo a García Nossa y al propio Fals Borda, cuyos postulados no tuvieron, a su juicio, mucha incidencia en el diseño de políticas públicas pero sí en la formación de nuevos analistas, fuertemente influidos por una ideología de izquierda, cuyas reflexiones sobre los modelos de desarrollo tuvieron mucha importancia en los años 70 . 
Según Machado (2004), las políticas de ajuste orientadas por los organismos internacionales, en los años 80 , llevaron al repliegue de buena parte de los investigadores de izquierda y a un mayor énfasis en la macroeconomía que en aspectos sectoriales, lo que se tradujo en un decaimiento de la producción académica en torno al problema agrario. De manera paralela, la agudización del conflicto y la irrupción del narcotráfico desviaron la atención hacia el estudio de la violencia, incrementándose los análisis de corte histórico y político. De acuerdo con este autor, el énfasis en la macroeconomía se consolidó en los años 90, década en la cual la producción académica estuvo marcada por el Consenso de Washington, mientras los estudios sobre el sector rural se centraron en la competitividad y el desarrollo institucional, dejándose atrás la preocupación por la redistribución de la propiedad y el desarrollo rural. No obstante, Machado (2004) destaca el surgimiento, en la década de los 90, de varios grupos que diversifican el panorama de la investigación rural en Colombia: los partidarios del libre comercio, los defensores de la protección de la agricultura, los campesinistas, los expertos en temas de violencia, los analistas del desplazamiento forzado y de los derechos humanos, los institucionalistas y neoinstitucionalistas y los medioambientalistas.

En las últimas décadas se destacan además dos Misiones orientadas al estudio del sector agropecuario y rural: la Misión de Estudios del Sector Agropecuario, llevada a cabo entre 1988 y 1990 bajo la coordinación de Jesús Bejarano y Albert Berry; y la Misión Rural, realizada entre 1997 y 1998, coordinada por Rafael Echeverri. La primera tuvo un enfoque fundamentalmente economicista, mientras la segunda abordó lo rural desde una perspectiva que trasciende lo agropecuario, integrando análisis sociales, culturales, políticos y ambientales.

El balance realizado por Machado (2004) a inicios del presente siglo lo lleva a concluir que, pese a la persistencia de un pensamiento fuertemente influido por políticas económicas internacionales, existe una renovación y diversificación de la temática 
en los estudios rurales, así como una tendencia investigativa más multidisciplinaria y transdisciplinaria, sin presentar ejemplos concretos de estas tendencias. No obstante, Machado considera que la presencia de la academia no ha sido estable ni acumulativa y ha carecido de un discurso propio, alternativo y bien fundamentado sobre el desarrollo rural. En síntesis, de acuerdo con su planteamiento, los problemas estructurales y coyunturales del sector agropecuario y rural en Colombia exigen un conocimiento que no se está generando adecuadamente en la actualidad. La revisión del conjunto de la obra "La academia y el sector rural", coordinada por Machado, muestra una investigación especialmente concentrada en temas productivos, colonización, conflicto armado y desplazamiento forzado.

Un análisis comparativo con las tendencias identificadas en América Latina permite observar con mayor claridad el panorama nacional. Kay (2007), cuyo análisis parte de la década de los 80 del siglo pasado, considera que las acciones colectivas de indígenas y mujeres rurales ha generado una explosión de estudios sobre género e indígenas que, sumados a las nuevas experiencias migratorias, han provocado un interés creciente en los estudios sobre la identidad y la cultura. Plantea así mismo que la crisis del socialismo y la extensión de las relaciones capitalistas han reducido la influencia de la teoría marxista en los estudios rurales, mientras los análisis estructuralistas han sido desplazados por los estudios sobre la agencia y las capacidades de los sujetos para crear sus propias estrategias de vida. Así mismo, Kay (2007) se refiere a la importancia que han tenido en las últimas tres décadas -aunque con distintos niveles de desarrollo- el estudio de las transformaciones en la economía y en la sociedad rural provocadas por la dinámica de la globalización neoliberal, los procesos de diferenciación campesina, la brecha entre la economía campesina y la empresarial, la feminización y la precarización del trabajo rural, las nuevas relaciones rural-urbanas, la reforma agraria, el surgimiento del movimiento campesino e indígena, la problemática de la pobreza rural, los impactos de los tratados de 
libre comercio sobre los campesinos y la seguridad alimentaria, las relaciones entre lo local y lo global, el desarrollo territorial rural y las políticas públicas, algunos de ellos en el marco de la denominada nueva ruralidad.

Bengoa (2003), en un análisis sobre los estudios rurales en América Latina en los últimos 25 años, destaca también un énfasis creciente en la dimensión étnica y de género y, en general, en los estudios sobre la cultura y la identidad. Según puede concluirse, pese a algunos puntos de encuentro, existen claras diferencias con las tendencias identificadas en el país, donde parece seguir existiendo un predominio de los estudios económicos, quizás sólo superado por los análisis sobre el conflicto armado y el desplazamiento forzado.

\subsection{Estudios en torno a las sociedades y economías campesinas}

Un campesino conversaba con un profesor de Universidad. Ante las agudas observaciones del campesino, el profesor ponía ojos como platos: "No se asombre usted. Los que no hemos estudiao no tenemos más remedio que hacer uso de la cabeza" (Ibáñez, 1991).

En coherencia con lo señalado por Fals Borda (1963) en torno a la sociología rural, un análisis realizado por Tocancipá (1998) sobre los estudios campesinos en la antropología colombiana entre 1940 y 1960, muestra que la emergencia de lo campesino en el marco de la antropología se acompaña de una preocupación más empírica que teórica: más que dar cuenta de lo campesino desde una perspectiva etnográfica y social, el papel de la antropología estuvo determinado desde una orientación instrumental, como consecuencia de la demanda del Estado sobre las disciplinas sociales, en su empeño de llevar a cabo el proyecto modernizador. Desde su punto de vista, aún persisten dificultades teóricas y metodológicas en los estudios sobre este grupo social: las investiga- 
ciones no han sido exhaustivas, se han circunscrito a situaciones locales y han carecido de continuidad, lo que se relaciona con los reducidos espacios de publicación, los tipos de investigación abordados y el escaso número de antropólogos dedicados al estudio de las sociedades campesinas. La poca atención de los antropólogos hacia los campesinos es explicada por Correa (2006) como una consecuencia de haber convertido a las poblaciones indígenas, prehispánicas o contemporáneas en dominio privilegiado del ejercicio de la antropología en Colombia, tendencia que había puesto en evidencia Friedmann en 1971, al llamar la atención sobre la necesidad de integrar en sus estudios a la población afrocolombiana y campesina.

Un análisis no circunscrito a esta disciplina sobre los estudios en torno al campesinado y a las economías campesinas es el realizado por Salgado (2004) como parte del proyecto Prospectiva del desarrollo rural y agrario para la paz en Colombia. Este autor llama igualmente la atención sobre la escasa producción académica y los pocos centros universitarios y de investigación con un trabajo permanente en este tema durante el período 1985-2002, aunque resalta la continuidad de los análisis de autores como Absalón Machado, Jesús Bejarano y Mariano Arango, así como el trabajo desarrollado por el Instituto de Estudios Rurales de la Universidad Javeriana, que además de los estudios sobre la nueva ruralidad y los sistemas sostenibles agrarios, hace un análisis continuo en la evolución de las economías campesinas por parte de un grupo de "campesinólogos", encabezado por Jaime Forero.

De acuerdo con Salgado (2004), el período analizado es más prolífico en aspectos relativos a la macroeconomía agraria y a los estudios sobre la violencia, los cuales, “... se detienen poco a estudiar al sujeto campesino en sus condiciones económicas, sociales y culturales" (105). Considera, por tanto, que el diseño y ejecución de políticas para los campesinos se está llevando a cabo sin una comprensión cabal de su evolución y de sus roles, al desconocer su mundo social y político y al basarse en una visión 
económica preconcebida y analíticamente precaria. No obstante, considera que en años recientes los pocos estudios realizados sobre las economías campesinas han utilizado un marco interpretativo renovado y optimista y han introducido una dimensión política orientada a comprender al campesino como sujeto social. En el campo económico, este investigador llama la atención, de manera particular, sobre los análisis realizados por Mariano Arango y Jaime Forero, cuyas conclusiones son, a su juicio, de suma importancia para discutir la viabilidad de las economías campesinas, al cuestionar con rigor el marco analítico de quienes trabajan desde una perspectiva productivista. Subraya, así mismo, algunos estudios de carácter sociológico, histórico y político, especialmente la contribución de Jaime Eduardo Jaramillo en la comprensión de la vida social y productiva de los campesinos, así como el fortalecimiento de los estudios sobre mujeres rurales.

Como parte del proyecto Prospectiva del desarrollo rural y agrario para la paz en Colombia, Mesías (2004) analiza el tema específico del campesinado y la democracia. Esta investigadora concluye que los campesinos han sido estudiados desde una perspectiva eminentemente económica y productivista, invisibilizando la importancia de su potencial político y limitando las posibilidades de comprensión de la ruralidad y del conflicto armado colombiano. Así mismo, identifica en la gran mayoría de trabajos académicos y en los discursos de gobierno del período analizado, mayor énfasis en la construcción formal de la democracia que en el análisis de lo que significa ésta, desde los sujetos históricos y desde los espacios locales.

Salgado (2004) llega a la conclusión de que no sólo existe una concepción convencional equivocada sobre el campesinado sino que la formulación de políticas públicas sobre el desarrollo rural se está llevando a cabo sin una comprensión adecuada de la problemática campesina: “En esta situación ha influido notoriamente un tipo de academia que abandonó el estudio del campesinado y definió, por cuenta de su vieja formación y de los procesos 
globales del desarrollo, que un amplio sector de población del campo no es útil en la acumulación contemporánea" (145).

Un esfuerzo importante por contrarrestar los vacíos identificados, tanto en el estudio de la ruralidad como del campesinado, lo constituye el reciente Informe Nacional de Desarrollo Humano: Colombia rural, razones para la esperanza, y la serie de Cuadernos que hacen parte de este Informe (PNUD, 2011), dedicados a analizar la situación específica de los campesinos, indígenas y mujeres rurales, así como la problemática del conflicto armado y el desplazamiento forzado.

\subsection{Estudios con jóvenes rurales}

La marginalidad de lo rural se pone en evidencia también en el estado del arte sobre jóvenes en Colombia (Escobar, 2004). Según se concluye en este estudio, la mayor parte de las investigaciones alude a jóvenes urbanos, existiendo una clara tendencia a invisibilizar las diversidades rurales y étnicas, y a privilegiar a los jóvenes de sectores populares urbanos, las organizaciones formales juveniles y, en muchos casos, el exotismo de una determinada agrupación juvenil.

El análisis de los ejes temáticos considerados -visiones de futuro, familia, cuerpo, educación, inserción sociolaboral, convivencia y conflicto, culturas juveniles / producción y consumo cultural, participación social y política y políticas públicasmuestra que la producción de conocimiento sobre los jóvenes rurales se centra fundamentalmente en dos de estos factores: "inserción sociolaboral" y "convivencia y conflicto". En relación con el primero, se destacan, de manera especial, las dinámicas de la vinculación de los jóvenes rurales en actividades laborales ilegales; mientras en el segundo caso se alude al conflicto social que afecta a la población rural juvenil -manifiesto en la ausencia de ofertas educativas, laborales y culturales- y a la vinculación de los jóvenes a algunos de los ejércitos armados que hacen parte del conflicto político del país (Escobar, 2004). 
En coherencia con lo anterior, de los principales matices en las nociones de sujeto rastreadas en las investigaciones -el sujeto vulnerable y en riesgo, el sujeto peligroso, el joven como motor del cambio social, el joven en búsqueda de identidad, el sujeto adscrito a culturas juveniles-, lo rural sólo es mencionado en relación con dos categorías: "sujeto vulnerable y en riesgo" y "sujeto peligroso". En relación con la primera categoría, se hace referencia al abandono del Estado y a la marginalización, fenómenos asociados nuevamente con la vinculación del joven a grupos armados o a cultivos ilícitos, aspectos a los que se suma, en investigaciones más recientes, el fenómeno del desplazamiento forzado. En cuanto a la segunda categoría, "sujeto peligroso", se hace referencia también a la vinculación de los jóvenes rurales a actividades relacionadas con los cultivos ilícitos y con el conflicto armado del país. Lo rural no es mencionado en las demás categorías, su ausencia es más que notoria en las aproximaciones: el joven como motor del cambio social, el joven en búsqueda de identidad, el sujeto adscrito a culturas juveniles.

Un estado del arte realizado específicamente sobre juventud rural (Jaramillo y Osorio, 2010), muestra también la invisibilización de los jóvenes rurales en los ámbitos académicos e institucionales, ocupando Colombia un lugar marginal en América Latina en relación con este tipo de estudios. Se identifica, así mismo, un abordaje coyuntural, carente de continuidad. Según este estudio, en los análisis centrados directamente en los jóvenes rurales sobresale el tema de la participación social y política, mientras en los estudios sobre lo rural, los jóvenes son invisibilizados, o bien, destacados por su participación en el conflicto armado, la migración a centros urbanos y el papel que desempeñan en la producción agropecuaria.

\section{2. ¿Tacto o indiferencia?}

Los estados del arte referidos, revelan la presencia de algunos autores comprometidos de manera amplia y continua con la 
comprensión de la vida campesina, pero a la vez, una exclusión relativamente generalizada de este grupo social en los estudios realizados en el país, así como una visión fragmentada, producida por una atención selectiva a algunas temáticas. La investigación actual parece ser más amplia -en número y en dimensiones analizadas- si se consideran los estudios sobre campesinos desplazados, pero no sobre quienes han resistido los embates del conflicto armado. No obstante, según se concluye a partir de la revisión realizada, las debilidades de la investigación social en torno a los campesinos colombianos no parece haber sido ocasionada por una reorientación de las preocupaciones de la academia hacia el desplazamiento forzado.

En general, puede considerarse que los investigadores sociales, salvo algunas excepciones, tienden a reproducir el carácter subsidiario de lo campesino y lo rural. Este hecho es inquietante, tanto como la fragmentación resultante de la atención selectiva a algunos ámbitos de análisis. Aunque Machado (2004) identifica en su estado del arte una diversificación reciente de las áreas de interés y un trabajo más interdisciplinario en torno al sector rural, tanto él como los demás autores revisados ponen en evidencia un fuerte énfasis en aspectos económicos, conflicto armado y desplazamiento forzado. Más que desconocer la importancia de estas dimensiones, preocupa el concepto de campesino que los investigadores sociales ayudan a construir y el ocultamiento de dimensiones clave de su constitución como sujeto: política, cultural, psicológica, educativa, social. El caso de los jóvenes es más que notorio, según se evidencia en los dos estados del arte antes reseñados, que muestran cómo la producción de conocimiento se centra, en este caso, en la inserción sociolaboral y en la vinculación al conflicto armado.

Estas tendencias quizás no son casuales. Distintos autores han llamado la atención sobre el papel desempeñado por las ciencias sociales en el proyecto de modernidad: "Sin el concurso de las ciencias sociales, el Estado moderno no se hallaría en la 
capacidad de ejercer control sobre la vida de las personas, definir metas colectivas a largo y a corto plazo, ni de construir y asignar a los ciudadanos una 'identidad' cultural" (Castro-Gómez, 2000, 3). De acuerdo con este mismo autor, "la invención del otro", entendida como el modo en que cierto grupo de personas se representa mentalmente a otras, surge del intento de crear perfiles de subjetividad estatalmente coordinados.

Según lo señalado antes, los primeros estudios realizados en relación con lo campesino y lo rural tuvieron una motivación esencialmente instrumental, condicionada en buena medida por organismos internacionales: la superación del "subdesarrollo". No puede considerarse, sin embargo, que se trate de una etapa inicial, sino más bien de una tendencia inherente a la producción científica convencional. En palabras de Fals Borda (1985, 136): “Toda ciencia, como producto cultural, busca un propósito humano determinado y, por lo mismo, leva implícitos los sesgos valorativos de las clases a las cuales pertenecen los científicos. En otras palabras, favorece a quienes la producen y controlan".

Ahora bien, ¿qué consecuencias tiene la actual tendencia de una escasa investigación sobre los campesinos en las posibilidades de transformación de sus condiciones de vida? Esta relación no parece tan clara. En un análisis sobre la posibilidad de resituar una perspectiva crítica que articule la investigación con la transformación social, Huergo (2002) considera que existen varias tendencias que la entrampan, una de las cuales es la tradición iluminista a partir de la cual se presenta la investigación como un insumo clave y previo para la toma de decisiones transformadoras de la sociedad y de la vida. A su parecer, "lo que esta tendencia contribuye a distorsionar es, por un lado, las razones históricas de los fracasos de los contratos sociales y, por el otro, los modos en que la obsesión por la 'armonía comunicacional' configura los posicionamientos de los investigadores" (37). Huergo ve necesario, por tanto, un desplazamiento de la política hacia lo político, que implica que los investigadores se reconozcan como 
sujetos de la crisis y la transformación y no sólo como observadores o intérpretes, dada la posibilidad de terminar por reforzar el divorcio entre investigación y sociedad.

El fracaso de la tradición referida por este autor se evidencia en lo sucedido en el país en relación con las dos Misiones de estudios rurales llevadas a cabo en las dos últimas décadas: la Misión de Estudios del Sector Agropecuario y la Misión Rural, cuyos aportes fueron desconocidos por los gobiernos de turno. Llama aún más la atención el caso de la Misión Rural, cuyas propuestas fueron discutidas no sólo con distintos actores sociales y políticos sino también con funcionarios del gobierno, y cuya financiación fue cubierta parcialmente con recursos públicos (Machado, 2004).

Esta situación conlleva necesariamente a la reflexión sobre las formas de abordaje de la investigación social, los intereses que la animan y la participación de los sujetos en la producción de conocimiento. Exige, por tanto, considerar los niveles de compromiso ético y político que suelen atribuirse a distintas perspectivas epistemológicas y enfoques de investigación. En este contexto cabe preguntarse: ¿qué formas de interacción entre campesinos e investigadores podrían considerarse moral y políticamente adecuadas?, ¿se encuentran estas formas de interacción necesariamente circunscritas a ciertas perspectivas epistemológicas y enfoques de investigación?

Las ciencias sociales de segunda mitad del siglo XX le reclamaron al positivismo su olvido intencional de los elementos éticos y políticos de la investigación. Pronto el énfasis se trasladó a las posibilidades de la interpretación y la intersubjetividad que abanderó la hermenéutica. No obstante, las investigaciones realizadas bajo esta última perspectiva son también debatidas por algunos autores, considerando, entre otros aspectos, la no consideración de los nexos entre conocimiento y poder, y el papel de los sujetos participantes en la investigación una vez registrado su discurso: la imposibilidad de éstos para realizar una apropiación de la reflexión generada durante el proceso y, salvo algunas 
excepciones, de cuestionar las interpretaciones del investigador (Torres, 2006; Cerillo, 2009). En este ámbito de análisis, la perspectiva crítica social suele plantearse como la alternativa que permite trascender la escasa participación de los sujetos en la construcción de conocimiento, propiciar la interacción de distintos saberes e integrar explícitamente las dimensiones ética y política en sus prácticas. No obstante, la reflexión sobre los aspectos éticos y políticos inherentes a la construcción de conocimientos sobre los campesinos o con los campesinos es mucho más compleja de lo que apenas hemos insinuado, no sólo por la posibilidad de discutir elementos éticos y políticos no circunscritos a tales perspectivas, sino también por la posibilidad de abordar esta reflexión por fuera del discurso estrictamente académico, considerando, entre otros aspectos, las propias perspectivas de los campesinos.

La información disponible en los estados del arte analizados es insuficiente para identificar tendencias claras respecto a las perspectivas epistemológicas a partir de las cuales han sido abordadas las investigaciones realizadas. No obstante, se pueden resaltar algunos casos paradigmáticos en la historia del país, en particular, las investigaciones de Orlando Fals Borda, Alfredo Molano y Jaime Forero. Fals Borda ha sido ampliamente reconocido por su compromiso ético y político con las comunidades campesinas. Su propuesta puede considerarse no sólo como la primera ruptura generada con las formas de investigar predominantes en los inicios de las ciencias sociales en el país, sino también como el esfuerzo más sólido por crear una ciencia alternativa, autónoma, que alcanzó su concreción en la Investigación Acción Participativa (IAP), definida por él como un "proceso que combina la investigación científica y la acción política para transformar radicalmente la realidad social y económica y construir el poder popular en beneficio de los explotados" (Fals Borda, 1985). A diferencia de lo que ocurre en otros enfoques, el establecimiento de una relación sujeto/sujeto no se limita, en la IAP, a ciertos momentos del proceso, promoviendo la participación desde la propia elección del objeto de investigación hasta la producción 
final y la divulgación de los hallazgos de los estudios. Se trata, así mismo, de un proceso cuyo plazo y ámbito no puede obedecer a una estricta planificación, por lo que se considera que la persistencia en todos los terrenos, y a largo plazo, es parte integral de esta propuesta (Fals Borda, 1985).

Si bien a esta perspectiva crítica suele atribuírsele un marcado compromiso ético y político con los sujetos de investigación, este mismo tipo de compromiso se encuentra en otros trabajos, por ejemplo, en los desarrollados por Molano y por Forero, desde perspectivas epistemológicas y metodológicas distintas. Los aportes de Molano -realizados desde una perspectiva comprensiva- son ampliamente reconocidos por sus contribuciones al conocimiento de la realidad social del país, sobre todo, en lo que se refiere a las problemáticas vividas en las zonas rurales -conflicto armado, desplazamiento forzado, violación de los derechos humanos, latifundio, narcotráfico-, rescatando las voces de los excluidos. Los trabajos de Forero, por su parte, son reconocidos por cuestionar los diagnósticos convencionales sobre la productividad y por mostrar la viabilidad de las economías campesinas. En reiteradas ocasiones -a partir de investigaciones abordadas desde una perspectiva empírico-analítica-, este autor ha defendido la viabilidad de estas economías, mostrando que las limitaciones de ingresos de los campesinos están ligadas a la falta de tierra y no a su supuesta ineficiencia (Forero, 2009). Así pues, la naturaleza de la relación entre investigadores y campesinos merece discutirse, así como la complejidad de las dimensiones ética y política inherentes a los procesos de investigación social, cuya expresión no parece circunscribirse, en definitiva, a una única perspectiva epistemológica.

Más allá de la indiscutible contribución de investigadores como los aquí citados -y de otros que han sido mencionados a lo largo del artículo-, los estados del arte analizados destacan importantes vacíos en los aportes que desde la investigación social se están realizando a los campesinos del país, contribu- 
yendo a la producción de no existencia de este grupo social, concepto utilizado por Santos (2006) para denotar la situación vivida por aquellos sujetos históricamente descalificados, tornados invisibles, ininteligibles o descartables de un modo irreversible. Lejos parecen estar estos vacíos del tacto reservado al que alude Heidegger.

\section{Referencias}

Baigorri, A. (1995). De lo rural a lo urbano. Hipótesis sobre las dificultades de mantener la separación epistemológica entre sociología rural y sociología urbana en el marco del actual proceso de urbanización global. Documento presentado en el V Congreso Español de Sociología, Grupo 5. Sociología Rural. Sesión $1^{\text {a }}$ : La Sociología Rural en un contexto de incertidumbre, Granada, España.

Bengoa, J. (2003). 25 años de estudios rurales. Sociologías, 10, 36-98.

Castro-Gómez, S. (2000). Ciencias sociales, violencia epistémica y el problema de la «invención del otro». En Lander, E. (compilador). La colonialidad del saber: eurocentrismo y ciencias sociales. Perspectivas Latinoamericanas. Buenos Aires: CLACSO.

Cerrillo, J. (2009). El intermediario imposible. Algunas reflexiones en torno a epistemología y ética en la investigación cualitativa. Nómadas. Revista Crítica de Ciencias Sociales y Jurídicas, 24, 187-201.

Correa, F. (2006). Interpretaciones antropológicas sobre lo indígena en Colombia. Universitas Humanística 62, 15-41.

Cubides, F. (1998). La Sociología en Colombia: demandas y tribulaciones. Revista de estudios sociales, 1, 9-15.

Escobar, M. (2004). Estado del arte del conocimiento producido sobre jóvenes en Colombia, 1985-2003. Informe final de investigación. Bogotá: Universidad Central, Departamento de investigaciones DIUC, Línea de investigación en Jóvenes y Culturas Juveniles.

Fals Borda, O. (1963). Desarrollo y perspectivas de la sociología rural en Colombia y en la América Latina. En Memoria del primer Congreso Nacional de sociología (153-172). Bogotá: Iqueima. 
Los investigadores sociales y su relación con los campesinos en Colombia: ¿tacto o indiferencia? - María Teresa Matijasevic y Alexander Ruiz Silva

Fals Borda, O. (1985). Conocimiento y poder popular. Lecciones con campesinos de Nicaragua, México y Colombia: estudio preparado para los grupos de base y para la Oficina Internacional del Trabajo. Bogotá: Siglo XXI Editores.

Forero, J. (2009). Un campo para el campo. Multimedia de la Revista Semana, noviembre 23 de 2009. Recuperado el 20 de mayo de 2010, de http:/ / www.semana.com/multimedia-problemas-sociales/campo-para-campo/2734.aspx

Friedemann, N. de (1971). Antropólogos y Antropología en Colombia: sus responsabilidades. Revista de la Dirección de Divulgación Cultural. Universidad Nacional de Colombia, 9, 5-16.

Heidegger, M. (1963). ¿Por qué permanecemos en la provincia? Traducción de Jorge Rodríguez. Revista Eco, Tomo VI, 5. Recuperado el 15 de marzo de 2010, de http: / / www.heideggeriana.com.ar/textos/en_provincia.htm

Huergo, J. (2002). Nuevas aventuras de la perspectiva crítica: la investigación "con" la transformación social. Revista Nómadas, $17,36-45$.

Ibáñez, J. (1991). Comunicaciones entre los pueblos y la ciudad. Política y Sociedad, 8, 95-100.

Jaramillo, O.E. y Osorio, F.E. (2010). Estado del arte juventud rural. Texto no publicado, $34 \mathrm{p}$.

Kay, C. (2007). Algunas reflexiones sobre los estudios rurales en América Latina. Iconos. Revista de Ciencias Sociales, 29, 31-50.

Llambí, L. (2010). ¿Hacia una sociología de los procesos territoriales? La transformación de los territorios rurales latinoamericanos a inicios del siglo XXI y los retos de la interdisciplinariedad. Documento presentado en el VIII Congreso Latinoamericano de Sociología Rural, Porto de Galinhas, Brasil.

Machado, A. (2004). La academia y el sector rural: sus vínculos, sus interpretaciones, sus retos. En Machado, Absalón (coordinador). La academia y el sector rural, volumen 1. Bogotá: Universidad Nacional de Colombia-CID.

Mesías, L. (2004). ¿Qué dice y qué silencia la academia sobre la democratización campesina? En Machado, Absalón (coordinador). La academia y el sector rural, volumen 1. Bogotá: Universidad Nacional de Colombia-CID.

PNUD (2011). Colombia Rural. Razones para la esperanza. Informe Nacional de Desarrollo Humano 2011. Bogotá. 
Rosas, G. (2003). A la memoria de Jesús Antonio Bejarano: la cuestión agraria y el desarrollo. Revista de Economía Institucional, 5 (8). 243-248

Salgado, C. y Mesías, L. (2003). La misión rural 1997-1998. Informe para el proyecto "Prospectiva de desarrollo rural y agrario para la paz en Colombia". Bogotá: CID/Universidad Nacional COLCIENCIAS.

Salgado, C. (2004). Economías campesinas. En Machado, Absalón (coordinador). La academia y el sector rural, volumen 1. Bogotá: Universidad Nacional de Colombia-CID.

Santos, B. de S. (2006). Conocer desde el Sur. Para una cultura política emancipatoria. Perú: Fondo Editorial de la Facultad de Ciencias Sociales.

Stavenhagen, R. (1965). Estado actual de la sociología rural en América Latina Foro internacional, 5 (4), 592-604.

Suárez, G. (2004). Crecimiento y desarrollo, la producción agropecuaria en Colombia 1986-2003. En Machado, Absalón (coordinador). La academia y el sector rural, volumen 4. Bogotá: Universidad Nacional de Colombia-CID.

Tocancipá, J. (1998). Los estudios campesinos en la antropología colombiana. 1940-1960. Revista Problemas Políticos Latinoamericanos, 3 (4-5), 171-205.

Torres, A. (2006). Por una investigación desde el margen. En Jiménez, A. y Torres, A. (compiladores). La práctica investigativa en ciencias sociales. Bogotá: Universidad Pedagógica Nacional. 